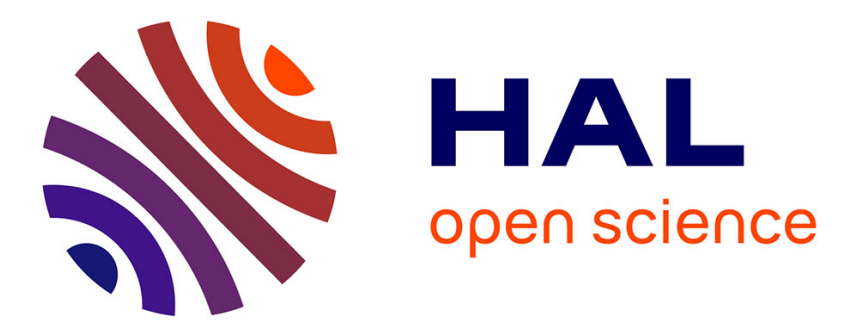

\title{
Semi-analytical Magnetic-Structural coupling with contact analysis for MEMS/NEMS
}

Phuong Pham Quang, Benoît Delinchant, Jean-Louis Coulomb, Bertrand Du Peloux

\section{- To cite this version:}

Phuong Pham Quang, Benoît Delinchant, Jean-Louis Coulomb, Bertrand Du Peloux. Semi-analytical Magnetic-Structural coupling with contact analysis for MEMS/NEMS. IEEE Transactions on Magnetics, 2011, 47 (5), pp.922-925. hal-00596833

\section{HAL Id: hal-00596833 \\ https://hal.science/hal-00596833}

Submitted on 30 May 2011

HAL is a multi-disciplinary open access archive for the deposit and dissemination of scientific research documents, whether they are published or not. The documents may come from teaching and research institutions in France or abroad, or from public or private research centers.
L'archive ouverte pluridisciplinaire HAL, est destinée au dépôt et à la diffusion de documents scientifiques de niveau recherche, publiés ou non, émanant des établissements d'enseignement et de recherche français ou étrangers, des laboratoires publics ou privés. 


\title{
Semi-Analytical Magneto-Mechanic Coupling With Contact Analysis for MEMS/NEMS
}

\author{
P. Pham Quang ${ }^{1,2}$, B. Delinchant ${ }^{1}$, J. L. Coulomb ${ }^{1}$, and B. du Peloux ${ }^{2}$ \\ ${ }^{1}$ Grenoble Electrical Engineering Laboratory, G2ELab, Saint Martin d'Heres 38400, France \\ 2 Cedrat SA, Meylan Cedex F-38246, France
}

This paper presents a methodology and a tool for magnetic and mechanical deformation coupling using numerical and analytical modeling. An analytical magnetic model using Coulombian approach is used and coupled with a mechanical deformation model for a cantilever beam to evaluate contact size and contact force. Such a coupling is not available using numerical solution. This paper details the deformation and contact analysis, which is validated by finite element simulation and also details the coupling approach. Such a modeling is dedicated to an optimization process of magnetic MEMS/NEMS in general and to magnetic nano switch in particular.

Index Terms-Contact analysis, magnetic switches, magneto mechanic coupling.

\section{INTRODUCTION}

M AGNETIC MEMS/NEMS have received much attention in recent years. This one gives a large actuation distance and requires a low voltage action compared with electrostatic MEMS/NEMS. One of the goals of MEMS/NEMS magnetic modeling (like magnetic nano switch [3], see Fig. 1) is the contact quality evaluation, which depends on surface and contact force.

No known tool can manage the coupling magneto-mechanic with contact analysis, that is the reason why the modeling of contact are often strongly approximated [1], [2]. In this paper, a new model and tool for semi-analytical magneto-mechanic coupling with contact analysis is presented. This tool is dedicated to the modeling and to the optimization process of MEMS/NEMS design. The optimization of a magnetic nano switch is presented as an application of our new methodology.

\section{MAGNeTiC Model}

\section{A. Magnet and Conductor Modeling}

Assumptions are constant magnetization of magnet and constant current density in conductor.

For magnet, Coulombian equivalent surface and volume charge approach is used

$$
\begin{aligned}
\sigma_{s} & =\vec{M} \cdot \vec{n}, \sigma_{v}=-\operatorname{div}(\vec{M}) \\
\vec{H}(\vec{r}) & =\iint_{v} \int_{v} \frac{\sigma_{v} \cdot\left(\vec{r}-\overrightarrow{r^{\prime}}\right)}{\left|\vec{r}-\overrightarrow{r^{\prime}}\right|^{3}} \cdot d v+\iint_{s} \frac{\sigma_{s} \cdot\left(\vec{r}-\overrightarrow{r^{\prime}}\right)}{\left|\vec{r}-\overrightarrow{r^{\prime}}\right|^{3}} d s
\end{aligned}
$$

\footnotetext{
Manuscript received May 31, 2010; accepted September 13, 2010. Date of current version April 22, 2011. Corresponding author: P. Pham-Quang (e-mail: phuong.pham-quang@g2elab.grenoble-inp.fr).

Color versions of one or more of the figures in this paper are available online at http://ieeexplore.ieee.org.

Digital Object Identifier 10.1109/TMAG.2010.2080662
}

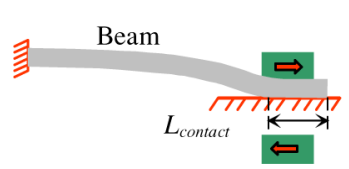

(a)

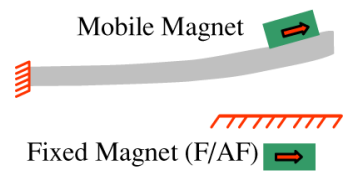

(b)
Fig. 1. Working principle of magnetic nano switch. (a) Closed state. (b) Open state.

For conductors, the Biot and Savart law is used

$$
\vec{H}(\vec{r})=\frac{1}{4 \pi}\left[\iint_{v} \frac{\vec{j} \times\left(\vec{r}-\vec{r}^{\prime}\right)}{\left|\vec{r}-\vec{r}^{\prime}\right|^{3}} d v\right] .
$$

These formulas (2) and (3) are integrated symbolically for some basic shapes and magnetization directions [4]-[6], leading to a full analytical magnetic field model, which allows fast and accurate calculations.

\section{B. Computing Forces and Magnetic Torques on Magnets}

For specific configurations such as interactions between parallelepiped magnets, the force can be fully analytical [5]. In our coupling, forces and magnetic torques applied on the barycenter of the beam are computed by adaptive numerical integrations, leading to a semi-analytical model

$$
\begin{aligned}
\vec{F} & =\iint_{s} \sigma_{s} \cdot \overrightarrow{H_{\mathrm{ext}}} d s+\iiint_{v} \sigma_{v} \cdot \overrightarrow{H_{\mathrm{ext}}} d v \\
\vec{\Gamma} & =\iiint_{v} \vec{M} \times \overrightarrow{H_{\mathrm{ext}}} d v .
\end{aligned}
$$

\section{Mechanical Deformation Model}

We have developed a model to compute deformation in the presence of contact of a cantilever beam with inputs and outputs as shown in Fig. 2.

The model is based on four hypotheses: 1) linear and isotropic materials; 2) small displacement-Bernoulli's hypothesis states: "Plane section remains plane after bending"; 3) neglecting the 


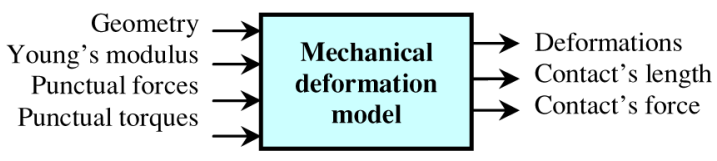

Fig. 2. Inputs and outputs of mechanical deformation model.

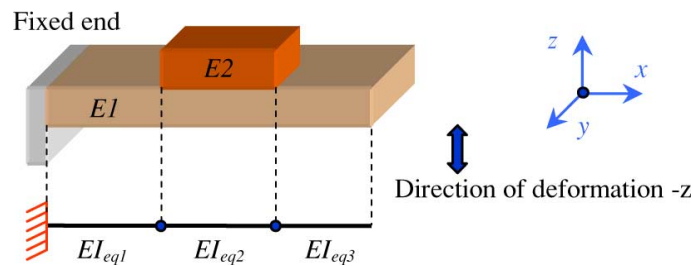

Fig. 3. Equivalent beam 1-D of beam in 3-D.

secondary deformations: considering only one direction of deformation; and 4) neglecting the friction on contact.

\section{A. Reducing the Beam in 3-D to 1-D}

Thanks to basic geometry of MEMS/NEMS, the beam consists of boxes. In addition, the last two hypotheses above which allow reducing the beam in 3-D to 1-D as shown in Fig. 3. Indeed, this reducing determines the $E I_{\text {eq }}$ of beam (equivalent product of $E$.I), where $E$ is Young's modulus (or elastic modulus) and $I$ is the second moment of area.

\section{B. Computing the Deformation of Beam 1-D}

Once the beam is reduced to 1-D, the equation used to compute the deformation is

$$
E I_{\mathrm{eq}} \cdot \partial^{2} z / \partial x^{2}=M
$$

where $z(x)$ and $M$ are, respectively, the deformation and the bending moment at the $x$-position.

The principle of superposition is used due to elastic linear deformation. Total deformation is equal to the sum of deformations created by each forces and torques

$$
z_{\text {total }}(x)=\sum_{\text {all_forces_and_torques }} z_{i}(x) .
$$

In addition, the total derivation of deformation is computed for contact analysis.

$$
d z(x)_{\text {total }}=\sum_{\text {all_forces_and_torques }} d z(x)_{i} .
$$

For each force or each torque, the bending moment is

$$
M=C_{1} \cdot x+C_{2}
$$

where $C_{i}$ are constants determined using adequate boundary conditions and connection conditions.

Solutions of (6) are

$$
\begin{aligned}
z_{i}(x) & =C_{1} \cdot x^{3} / 6+C_{2} \cdot x^{2} / 2+C_{3} \cdot x+C_{4} \\
d z(x)_{i} & =C_{1} \cdot x^{2} / 2+C_{2} \cdot x+C_{3} .
\end{aligned}
$$

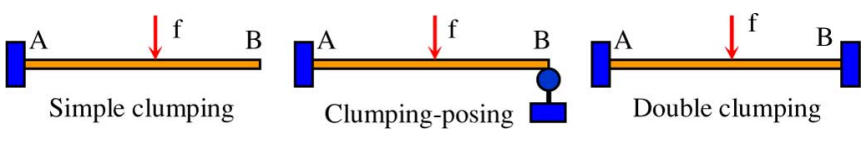

Fig. 4. Three types of beam categorized based on support conditions.

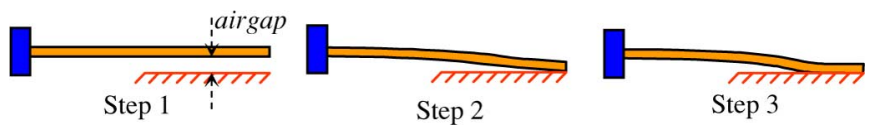

Fig. 5. Three steps of contact analysis.

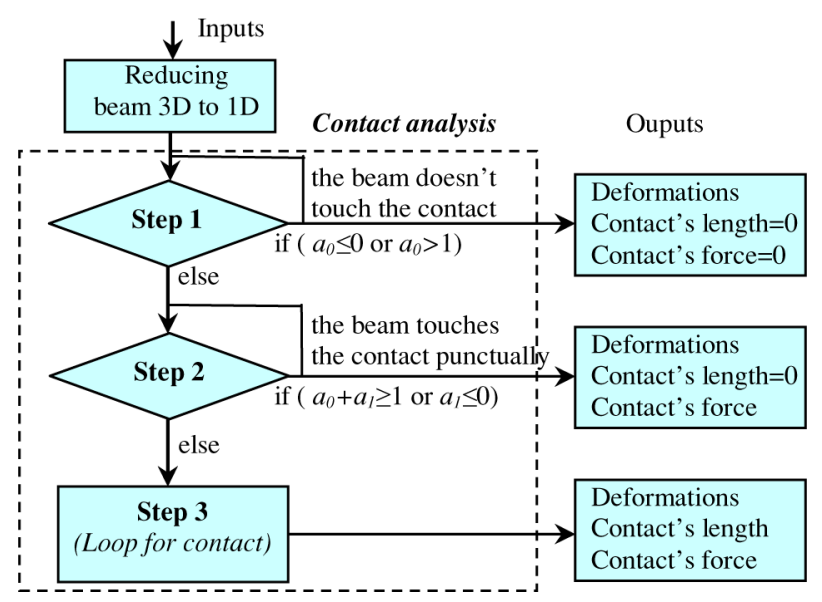

Fig. 6. Algorithm of contact analysis.

Connection conditions are continuous conditions of deformation between the beam pieces (with different $E I_{\mathrm{eq}}$ ).

Boundary conditions depend on beam type as Fig. 4.

\section{Contact Analysis}

Contact analysis is realized by decomposing in three steps, which can be superposed as shown in Figs. 5 and 6.

1) Step 1: In this step, we calculate $a_{0}$, which is the proportion of total forces, and torques for the beam touching the contact

$$
a_{0}=\operatorname{airgap} / z_{1-B}
$$

where $z_{1_{-} B}$ is deformation at the end of beam (point $\mathrm{B}$ ) with all forces and torques, with simple clumping beam. If $a_{0}=1$, the beam is just touching the contact.

2) Step 2: In this step, we calculate $a_{1}$ for which $\left(a_{0}+a_{1}\right)$ is the proportion of total forces and torques for a deformation's derivative equal to zero at the end of the beam

$$
a_{0} \cdot d z_{1_{-} B}+a_{1} \cdot d z_{2-B}=0
$$

where $d z_{1-B}$ is deformation's derivative at the end of the beam with all forces and torques with simple clumping beam, $d z_{2} B$ is the same with clumping-posing beam.

3) Step 3: In this step, the beam touches the contact and has a contact surface. 


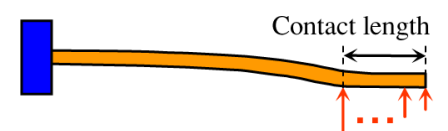

$\mathrm{m}$ punctual forces replacing contact

Fig. 7. Contact replaced by a distribution of force.

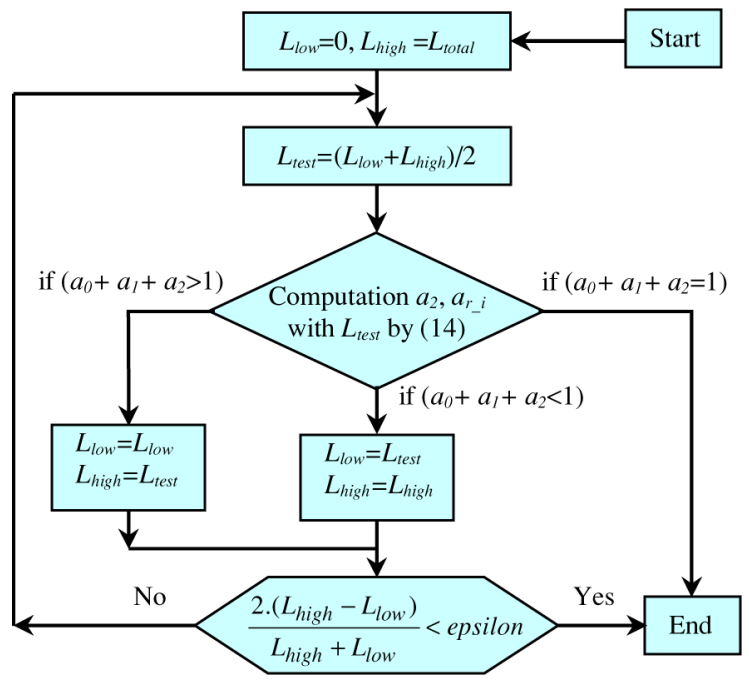

Fig. 8. Iterative method to compute the contact's length.

The contact is replaced by a distribution of $m$ forces $(m=$ $1,2, \ldots)$ as Fig. 7.

To find the forces and torques which are able to create a given length of contact, a linear system of $(m+1)$ equations with $(m+1)$ unknowns $\left(a_{2}, a_{r_{-} i}, i=1: m\right)$ has to solved

$$
\left\{\begin{array}{l}
a_{0} \cdot z_{1 \_L 1}+a_{1} \cdot z_{2 \_L 1}+a_{2} \cdot z_{2 \_L 1} \\
\quad+\sum_{i=1}^{m} a_{r_{-} i} \cdot z_{2 i r_{-} L 1}=\text { airgap } \\
\quad \ldots \\
a_{0} \cdot z_{1 \_L m}+a_{1} \cdot z_{2 \_L m}+a_{2} \cdot z_{2 \_L m} \\
\quad+\sum_{i=1}^{m} a_{r_{-} i} \cdot z_{2 r_{-} L m}=\text { airgap } \\
a_{0} \cdot d z_{1 \_L m}+a_{1} \cdot d z_{2 \_L m}+a_{2} \cdot d z_{2 \_L m} \\
\quad+\sum_{i=1}^{m} a_{r_{-} i} \cdot d z_{2 r_{-} L m}=0
\end{array}\right.
$$

where

- $\left(a_{0}+a_{1}+a_{2}\right)$ is the proportion of total forces and torques to create the given length of contact $L_{\text {contact }}$;

- $a_{r_{-} i}(i=1: m)$ is the $i$ th force value replacing the contact;

- $L_{i}=L_{\text {total }}-L_{\text {contact }} \cdot(m-i+1) / m(i=1: m)$ is the application point of $i$ th force replacing contact. $L_{\text {total }}$ is total length of beam;

- $z_{1 \_L i}, d z_{1 \_L i}(i=1: m)$ are, respectively, deformations and its derivative at $L_{i}$ with all forces and torques, with simple clumping beam;

- $z_{2 \_L i}, d z_{2-L i}(i=1: m)$ are, respectively, deformations and its derivative at $L_{i}$ with all forces and torques, with clumping-posing beam;

- $a_{r_{-} i} . z_{2 i r_{-} L k}, a_{r_{-} i} . d z_{2 i r_{-} L k}(i, k=1: m)$ are deformations and its derivative at $L_{k}$ with the $i$ th force replaced the contact, with clumping-posing beam.

As the distribution of force, which replaces the contact, is unknown, the length of contact cannot be founded directly. An iterative method detailed in the algorithm at Fig. 8 is used.

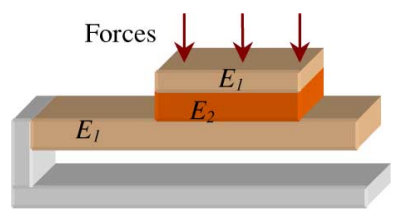

Fig. 9. Beam's geometry for validation of mechanical deformation model.

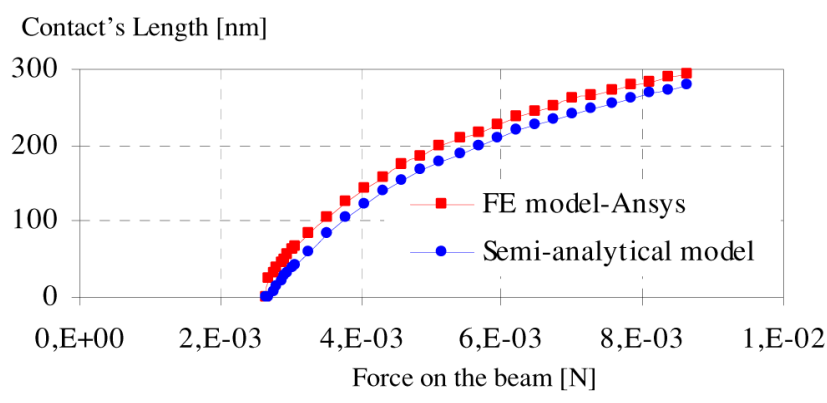

Contact's Force $[\mathrm{N}]$

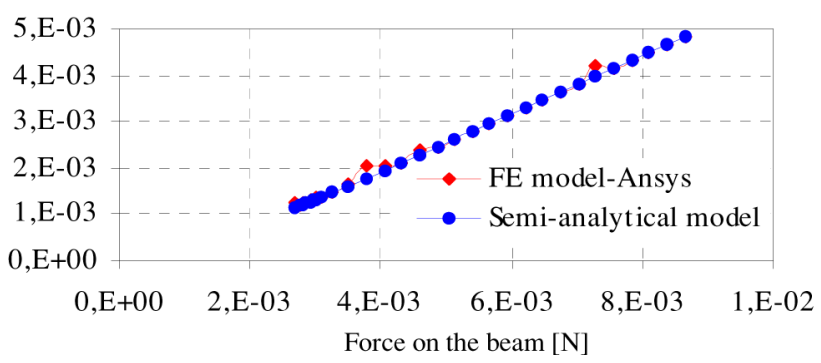

Fig. 10. Results of validation of mechanical deformation model.

The contact's force is computed by $F_{\text {Contact }}=F_{\text {total }}-$ $F_{\text {reaction_ } A}$, where $F_{\text {total }}$ is the total force applied on the beam, $F_{\text {reaction } \_A}$ is the reaction force on the fixed side of the beam (point A).

\section{Validation by Finite-Element Simulation}

Our mechanical deformation model has been validated by ANSYS software. The beam's geometry used is shown in Fig. 9. Three forces are applied on the beam.

Fig. 10 shows the variation of the contact length and contact force as function of forces applied on the beam. The results obtained by our model are closed to the results of finite-element simulation.

\section{Magneto-Mechanical Coupling}

A tool following as [4] has been developed to generate automatically the equations, which describe the sequential magneto-mechanic coupling.

The interaction between two fields is accomplished via the load vectors of forces, torques and positions as Fig. 11. The magnetic model requires the deformation to compute forces and torques. These forces and torques are used in the mechanical deformation model to compute positions of the beam. This coupling is equivalent to solve a system of two vector variables (two fields) with Gauss method. 


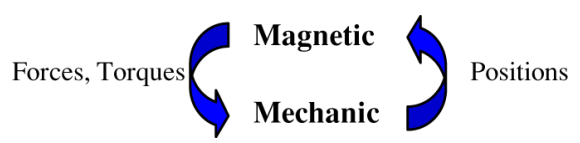

Fig. 11. Sequential magnetic-mechanical coupling.

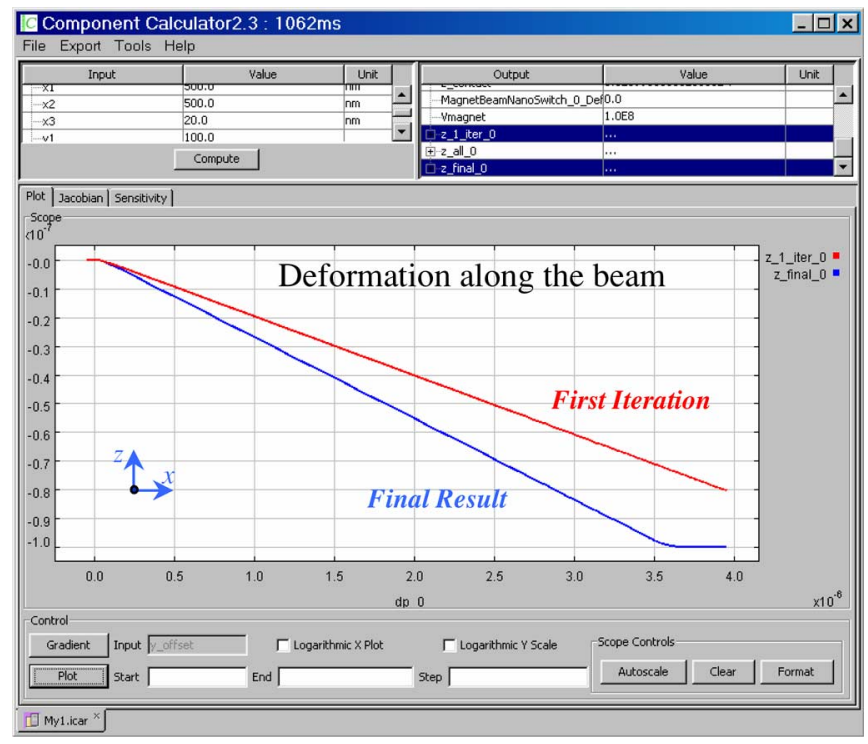

Fig. 12. Screenshot of our simulation tool visualizing the deformation along the beam of the first iteration and the final result.
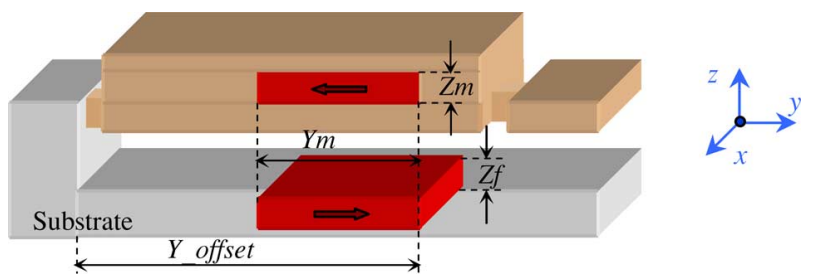

Fig. 13. Geometry and parameters to be optimized of a magnetic nano switch.

\section{Application: Magnetic NANo Switch Optimization}

\section{A. Working Principle}

Magnetic nano switches have many applications as power switches, nanomechanical memory ... Their working principle (as Fig. 1) is based on the deformation of a beam with magnet under the influence of the field created by a ferromagnetic/antiferromagnetic (F/AF) association, which is able to create more than $2 \mathrm{~T}$ fields without power supply [7]. The switching is achieved by reversing the magnetization of the F/A using conductors.

\section{B. Model}

In static, the association F/AF is as magnet. Consequently, our coupling tool is used to evaluate the quality of contact in closed state and to evaluate the deformation in open state.

The convergence is achieved with relative tolerance of $10^{-4}$ after some iterations. Fig. 12 shows the deformation along the beam of the first iteration and the final result.
TABLE I

OPTIMIZATION RESULTS OF MAGNETIC NANO SWITCH

\begin{tabular}{|l|l|l|c|c|}
\hline \multicolumn{1}{|c|}{ Inputs } & unit & Type of constraint & Initial values & Final values \\
\hline$Y m$ & $\mathrm{~nm}$ & {$[100 ; 800]$} & 500 & 230 \\
\hline$Z m$ & $\mathrm{~nm}$ & {$[50 ; 500]$} & 200 & 212 \\
\hline$Z f$ & $\mathrm{~nm}$ & {$[50 ; 500]$} & 200 & 222 \\
\hline$Y$ offset & $\mathrm{nm}$ & {$[2500 ; 3500]$} & 3200 & 3500 \\
\hline Outputs & & & & \\
\hline$L_{\text {contact }}$ & $\mathrm{nm}$ & $\geq 300$ & 313,71 & 310,86 \\
\hline$F_{\text {contact }}$ & $\mathrm{N}$ & $\geq 1,0 \mathrm{E}-8$ & $1,03 \mathrm{E}-8$ & $1,0001 \mathrm{E}-8$ \\
\hline$V_{\text {magnet }}$ & $\mathrm{nm}^{3}$ & To be minimized & $1,0 \mathrm{E} 8$ & $4,991 \mathrm{E} 7$ \\
\hline
\end{tabular}

\section{Optimization Specifications}

Geometry and parameters to be optimized are given in Fig. 13. The optimization aims to determine dimensions of both fixed and mobile magnets and their positions on beam, to minimize the volume of magnets, while respecting constraints such as length of contact and contact force to ensure the quality of contact or contact resistances below a desired value in closed state.

\section{Optimization Results}

The following results, as in Table I, have been obtained by a genetic algorithm, which can only be applied on fast models.

\section{CONCLUSION}

This paper presented a semi-analytical mechanical deformation with contact analysis model for the cantilever beam and the sequential coupling with magnetic model. This kind of model was not available before. Moreover, it is fast enough to the optimization process.

As no known numeric tool can manage the magnetic-mechanical coupling with contact analysis, each model has been validated separately by finite element method. Experimental validation is not yet available due to nanometric fabrication issues.

This work is supported by the French research agency (ANR, Monaco project) in order to simulate forces exerted on nanometric bodies. Our tool gives the user the ability to add equations, which is very useful to add complementary forces from van der Waals or Casimir effects.

\section{REFERENCES}

[1] G. D. Gray, Jr. and P. A. Kohl, "Modeling and performance of a magnetic MEMS wiping actuator," J. Microelectromech. Syst., vol. 15, no. 4, pp. 904-911, Aug. 2006.

[2] G. D. Gray and P. A. Kohl, "Magnetically bistable actuator. Part I, Part II," Sens. Actuators A, Phys., vol. 119, pp. 489-511, 2005.

[3] L. Bilhaut, P. Andreucci, L. Duraffourg, and B. Viala, "Nano-commutateur magnétique bistable," U.K. 08 52061, Mar. 28, 2008.

[4] H. L. Rakotoarison, B. Delinchant, and O. Cugat, "Methodology and tool for generating semi-analytical models used to pre-design electromagnetic MEMS," presented at the CEFC, Miami, FL, 2006.

[5] G. Akoun and J. P. Yonnet, "3D analytical calculation of the forces exerted between two cuboidal magnets," IEEE Trans. Magn., vol. Mag-20, no. 5, pp. 1962-1964, Sep. 1984.

[6] H. L. Rakotoarison, J. P. Yonnet, and B. Delinchant, "Using coulombian approach for modeling scalar potential and magnetic field of a permanent magnet with radial polarization," IEEE Trans. Magn., vol. 43, no. 4, pp. 1261-1264, Apr. 2007.

[7] J. Sort, B. Dieny, and J. Nogués, "Exchange bias in antiferromagneticferromagnetic-antiferromagnetic structures with out-of-plane magnetization," Phys. Rev. B, vol. 72, no. 10, 2005, 104412. 